\title{
Activation energy for the decay of two-dimensional islands on $\mathrm{Cu}(100)$
}

\author{
Christian Klünker, James B. Hannon,* Margret Giesen, and Harald Ibach $^{\dagger}$ \\ Institut für Grenzflächenforschung und Vakuumphysik, Forschungszentrum Jülich, D-52425, Jülich, Germany \\ Ghyslain Boisvert and Laurent J. Lewis \\ Département de Physique et Groupe de Recherche en Physique et Technologie des Couches Minces (GCM), Université de Montréal, \\ Case Postale 6128, Succursale Centre-Ville, Montréal, Québec, Canada H3C 3J7
}

(Received 18 March 1998; revised manuscript received 6 July 1998)

\begin{abstract}
Experimental data on the decay rate of two-dimensional islands on $\mathrm{Cu}(100)$ as a function of temperature are reported. The decay is limited by the attachment-detachment process. A comparison of the experimentally observed activation energy for the decay rate with results from first-principles theory renders further support to the understanding that on $\mathrm{Cu}(100)$ island decay is due to mass transport via vacancies.
\end{abstract}

[S0163-1829(98)50836-3]

The coarsening of two-dimensional (2D) islands on surfaces has been studied quite extensively in the past. On metal surfaces, different mechanisms for the coarsening process have been found to be operative. One is the classical mechanism of Ostwald ripening ${ }^{1}$ in which islands of larger size gain atoms at the expense of smaller ones. The thermodynamic driving force for the ripening process is the larger chemical potential of smaller islands. Akin to Ostwald ripening is the decay of islands in the vicinity of steps in which islands lose atoms to the ascending step edges. On metal surfaces where steps are abundant even on well-prepared surfaces, this process of island decay effectively competes with the Ostwald ripening, in particular at later times in the coarsening process. Another important coarsening mechanism is a consequence of the surprisingly large mobility of islands, vacancy islands, and steps on surfaces. ${ }^{2-7}$ Because of the high mobility, the small islands generated by homogeneous nucleation engage in a random walk on surfaces, meet occasionally, and coalesce. For the $\mathrm{Cu}(100)$ and the $\mathrm{Ag}(100)$ surface this random-walk-induced coalescence can be the prevailing mechanism in the coarsening process. ${ }^{8-10}$

Until recently it was tacitly assumed that atom exchange between islands and steps on metal surfaces is mediated via adatoms on the terraces as the diffusing species. In a recent study of Ostwald ripening on $\mathrm{Cu}(100)$ it was proposed that single atom vacancies rather than adatoms are responsible for the mass transport between the islands. ${ }^{11}$ The argument was based on the time dependence of the island decay that called for an activation energy for the attachment of the diffusing species to an island. As it is rather difficult to envision an activation barrier for the attachment of adatoms to an ascending step on a metal surface but quite natural to assume a barrier for the attachment of vacancies, vacancies were proposed to be the prevailing mass transport carrying species on $\mathrm{Cu}(100)$. A recent theoretical study ${ }^{12}$ has indeed shown that the activation energy for diffusion of vacancies on $\mathrm{Cu}(100)$ is smaller than for adatoms, rendering further support for the model of vacancy mediated coarsening on this surface. In this paper we report on further experimental and theoretical evidence for the vacancy mass transport mechanism. The evidence is based on a comparison of the activation energy for the decay rate of islands on $\mathrm{Cu}(100)$ to results of firstprinciples calculations of the energy of vacancy formation and diffusion.

The scanning tunneling microscope (STM) is based on the Besocke design, ${ }^{13}$ and is capable of variable-temperature operation in the range of $50-500 \mathrm{~K}$. The UHV chamber is equipped with an electron-beam evaporator for copper deposition. Special care was taken to degas the evaporator and the $\mathrm{Cu}$ source so that during deposition the pressure in the chamber never exceeded $1 \times 10^{-10}$ mbar.

The $\mathrm{Cu}$ single crystal was cut by spark erosion and polished mechanically to an accuracy of $0.1^{\circ}$. Its sulfur content was leached by heating in a 1:25 hydrogen and argon atmosphere at $800{ }^{\circ} \mathrm{C}$ for several hours prior to mounting in the UHV chamber. The in situ preparation consisted of sputtering for 20 min with $500 \mathrm{eV} \mathrm{Ne}{ }^{+}$ions, followed by 10 -min annealing periods at $1100 \mathrm{~K}$. After each annealing cycle, the sample was cooled slowly to room temperature. No contamination was detectable in the Auger spectrum after a few cycles. Since island decay is rather sensitive to contamination, a surface coverage below the detection limit of Auger spectroscopy may still have a non-negligible influence on the measurements. We therefore continued the cleaning procedure many cycles beyond the point at which we found the sample clean by Auger standards. The final state of the surface after the preparation procedure was controlled by means of the STM images. Final annealing of the sample prior to the deposition of copper involved heating to $800 \mathrm{~K}$ and a slow cooling at a rate of $0.5 \mathrm{~K} / \mathrm{s}$. After this procedure, the mean terrace width was about $100 \mathrm{~nm}$. In order to minimize thermal drift in the STM images the sample thermally equilibrated for approximately $30 \mathrm{~min}$ prior to deposition of $\mathrm{Cu}$. The remaining temperature drift was compensated by applying an additional bias to the piezoactuators.

For a convenient study of island decay, small $\mathrm{Cu}$ islands were evaporated on a surface on which large islands had been created already by evaporation at a higher substrate temperature. We therefore study the decay of small islands in an environment of large ones, a situation that differs from the work of Pai et al. ${ }^{9}$ The images were recorded using a tunneling current of $1.0 \mathrm{nA}$ with the tip biased negatively by 0.10 

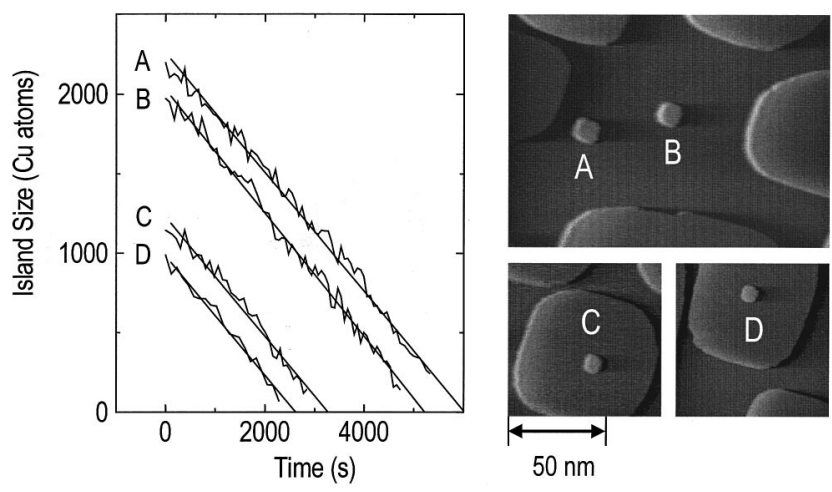

FIG. 1. Sections of STM images showing two sets of islands. One (islands A and B) placed in an environment of ascending steps of larger islands, the second (islands $\mathrm{C}$ and D) placed on top of another larger island. For all islands the final decay rate is independent of time and also independent of the environment, which is indicative of a detachment limited decay. The temperature was 343 $\mathrm{K}$.

$0.70 \mathrm{~V}$ with respect to the sample. The scan width was calibrated by means of atom-resolved images of the $\mathrm{Cu}(100)$ surface. The experimental data, in the form of island sizes as a function of time, were obtained in repeated scans of a fixed area of the surface (typically $150 \times 150 \mathrm{~nm}$ ) in intervals of 1-2 min. In order to analyze the STM images we used a special purpose computer code that allowed the simultaneous evaluation of the sizes of all islands in an image. The program fits a spline to the gray values of each scan line and searches for the largest slopes in this spline. Unphysical large jumps in the island edge due to noise are filtered out. In order to test the scaling of the areas found by the program we have recorded images of islands with different scan widths and evaluated the island area using the program. The areas of the islands in pixels as determined by the program were then compared to the areas expected from the nominal scaling of the STM. No deviation was found.

The decay of islands is either limited by atom transfer from the island to the terrace (detachment limit) or by transport across the terrace to another ascending step (diffusion limit). In the detachment limit the area $A$ of an island decays at a rate of

$$
\frac{d A}{d t}=-2 \pi \Omega^{2} \nu s \rho_{\infty} \frac{\tilde{\gamma}}{a k_{B} T} .
$$

Here $\Omega$ is the area of one atom, $a$ its diameter, and $k_{B}$ the Boltzmann constant. The hopping frequency $\nu$ characterizes the diffusion on the terrace. With this notation we assume that the diffusing species jumps only by one atom distance at a time. For a more general formulation, $\nu \Omega$ can be replaced by the diffusion coefficient in Eq. (1). The equilibrium concentration of the diffusing species per atom area and the line tension of the perimeter of the island are denoted as $\rho_{\infty}$ and $\tilde{\gamma}$, respectively. The island decay is in the detachment limit if the sticking coefficient $s$ [Eq. (1)] for the attachment of the diffusing species to the island is small $(s \ll 1)$. Equation (1) holds for the final stage of the life of an island, when the chemical potential of the island is large compared to the chemical potential of the diffusing species on the terrace around the island. In the detachment limit, the final decay

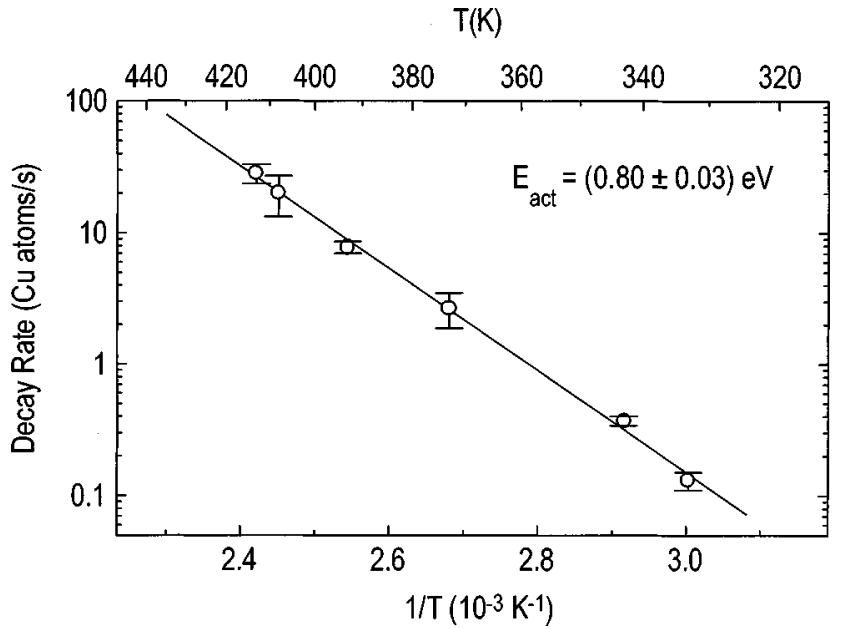

FIG. 2. Arrhenius plot of the decay rate near the end of the life of the islands. The activation energy is $0.80 \pm 0.03 \mathrm{eV}$.

rate is constant with time and does not depend on the environment of the decaying island. These two feature are characteristic of the detachment limited decay.

If the sticking coefficient $s$ is near unity, the transport of the diffusing species on the terraces is the limiting factor in the decay process. In that case, the decay rate always depends on the environment of the island. An analytical solution for the decay rate exists for the special case of an island with radius $R_{i}$ in the center of a vacancy island of radius $R_{v}$ :

$$
\frac{d A}{d t}=-2 \pi \Omega^{2} \nu \rho_{\infty} \frac{\tilde{\gamma}}{k_{B} T} \frac{1}{\ln \left(R_{v} / R_{i}\right)}\left(\frac{1}{R_{i}}+\frac{1}{R_{v}}\right) .
$$

In general, the decay rate can be obtained from a numerical solution of the diffusion problem in which the perimeters of the islands determine the boundary conditions. ${ }^{14}$ As seen from Eq. (2) the rate increases as the area approaches zero and becomes infinite at $A=0$. This and the dependence of the decay rate on the environment are characteristic features of the diffusion limited decay.

Examples of the final decay of islands on $\mathrm{Cu}(100)$ are presented in Fig. 1. The islands A and B are placed between the ascending steps of neighboring larger islands while the islands C and D sit on top of another island. Both set of islands decay with the same constant rate. The constant decay rate and the independence of the rate on the environment are indicative of a detachment limited island decay [Eq. (1)].

We note that the islands in Fig. 1 have the shape of rounded squares. This is the typical equilibrium shape of islands on a (100) surface. ${ }^{15}$ The island shape becomes more circular as the temperature increases. The observed island shape is roughly consistent with the analytical expression of the equilibrium shape in the Ising model when the kink energy of $\varepsilon=0.129 \mathrm{eV}$ (Ref. 16) is used. The thermodynamic theory of island decay used for the derivation of Eq. (1) assumes that the islands maintain their equilibrium shape during the decay. Evidence that this condition is met is available from experimental observations: Two-dimensional islands engage in a random walk across the surface because of the diffusion of atoms along the perimeter of the island. As a consequence of the random walk, every once in a while an island coalesces with another. The time scale necessary for 


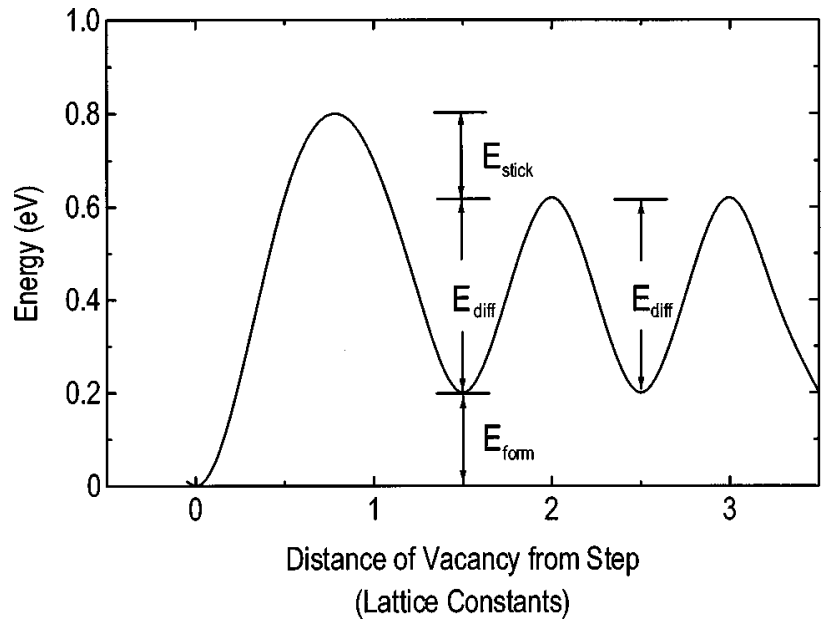

FIG. 3. Potential for the attachment of a vacancy to a kink in a step.

establishing the new equilibrium shape of the combined island was found to be very short compared to the decay time.

The rate of the final decay of islands was measured for temperatures between 330 and $415 \mathrm{~K}$. The result is plotted in Fig. 2 in an Arrhenius plot. We note that the decay rate at room temperature is rather low $(0.01$ atom/s). This slow rate is consistent with the observation that at room temperature coarsening proceeds only via island diffusion. ${ }^{9}$ The activation energy is found to be

$$
E_{\text {act }}=0.80 \pm 0.03 \mathrm{eV} \text {. }
$$

Three quantities in Eq. (1) involve activated processes: the hopping frequency, the sticking coefficient, and the equilibrium concentration. The equilibrium concentration of the diffusing species is

$$
\rho_{\infty}=e^{-E_{\text {form }} / k_{B} T},
$$

in which $E_{\text {form }}$ is the energy to generate the species from a kink in a straight step. Hence the experimental value of the activation energy $E_{\text {act }}$ is equal to the sum (Fig. 3)

$$
E_{\text {act }}=E_{\text {stick }}+E_{\text {diff }}+E_{\text {form }} .
$$

Because of the time dependence, the decay must be detachment limited. Hence $E_{\text {stick }}$ must be larger than $k_{B} T$ and the sum of the activation energy for diffusion and formation is smaller than $0.80 \mathrm{eV}$.

The static $(0 \mathrm{~K})$ activation energies for adatom and vacancy diffusion on $\mathrm{Cu}(100)$ have been calculated within both the semiempirical embedded-atom method ${ }^{17}$ (EAM) and the $a b$ initio density-functional theory (DFT), ${ }^{18}$ and the results are reported in Ref. 12. Further calculations have been carried out in order to determine the DFT formation energies; the results of these and previous calculations are summarized in Table I. We give here a brief overview of the computational approach; full details of the calculations, as well as a thorough discussion of the diffusion energies in the light of experiment and other calculations, can be found in Ref. 12 . The EAM study is based on the formulation of Foiles, Baskes, and Daw. ${ }^{17}$ The barriers were calculated using a slab
TABLE I. Diffusion ( $\left.E_{\text {diff }}\right)$ and formation $\left(E_{\text {form }}\right)$ energies (in $\mathrm{eV}$ ) for adatoms and vacancies on $\mathrm{Cu}(100)$ with both the EAM and the GGA of density-functional theory. (See the text for details and references.) The diffusion energies have been published in Ref. 12 and the EAM formation energies in Ref. 27.

\begin{tabular}{lcccccc}
\hline \hline Energy & \multicolumn{2}{c}{$E_{\text {diff }}(\mathrm{eV})$} & \multicolumn{2}{c}{$E_{\text {form }}(\mathrm{eV})$} & \multicolumn{2}{c}{$E_{\text {diff }}+E_{\text {form }}(\mathrm{eV})$} \\
\hline Method & GGA & EAM & GGA & EAM & GGA & EAM \\
Adatom & 0.52 & 0.50 & 0.48 & 0.71 & 1.00 & 1.21 \\
Vacancy & 0.42 & 0.47 & 0.22 & 0.59 & 0.64 & 1.06 \\
\hline \hline
\end{tabular}

consisting of 8 full layers, each containing 64 atoms (excluding the adatom or the vacancy). The bottom two layers were held fixed in order to mimic the presence of bulk material, and periodic boundary conditions were applied along the two lateral directions. For each case, the system was relaxed using a steepest-descent algorithm until the forces became negligible. For the DFT calculations, the pseudopotential-planewave approach was used, ${ }^{19}$ where the core orbitals are replaced by pseudopotentials, here of the Troullier-Martins form. ${ }^{20}$ We examined both the local-density approximation $^{21,22}$ (LDA) and the generalized-gradient approximation $^{23}$ (GGA) for the exchange-correlation energy. Results were found to be more accurate in the GGA, as is known to be the case for $3 d$ metals, ${ }^{24,25}$ and thus only the GGA results are discussed here. The surface was modeled, again, as a slab, and convergence studied with respect to lateral dimensions, number of layers, and Brillouin-zone sampling. The results for the adatom diffusion energy were found to be essentially converged for a four-layer, $3 \times 3$ system and we therefore used this geometry in the present calculations. One remarkable result shown in Table $\mathrm{I}$ is the close similarity between GGA and EAM for the diffusion barriers for both adatoms and vacancies. ${ }^{12}$ Such an agreement was observed, also, for dimer exchange (EAM $0.74 \mathrm{eV}$, GGA $0.79 \mathrm{eV}$ ) and, to a lesser extent, adatom exchange (EAM $0.73 \mathrm{eV}$, GGA $0.96 \mathrm{eV}$ ), though evidently differences are sizable and significant since processes are activated. The calculated activation energies for diffusion are higher than values determined experimentally. ${ }^{26}$ Experimental values were obtained, however, by indirect methods, e.g., by making certain assumptions about the nucleation process. The "experimental" numbers are therefore not necessarily more accurate than the theoretical results. Concerning formation energies, the EAM values have been published by Karimi et al., ${ }^{27}$ and are listed in Table I along with the DFT-GGA results of the present study. We find that EAM and DFT are in disagreement in this case, which is a bit surprising in view of the excellent accord for diffusion barriers discussed above. It should be mentioned that the DFT-GGA values have some uncertainties associated with them (arising from finite-size limitations), that we estimate to be of at most 0.1 eV. ${ }^{12}$ Also given in Table I are the sums of $E_{\text {diff }}$ and $E_{\text {form }}$ for both the adatom and the vacancy; this sum should be a lower bound to the total activation energy $E_{\text {act }}$, which, according to Eq. (5), includes a (nonzero) sticking energy $E_{\text {stick }}$. Assuming that our calculated values are correct, only vacancy diffusion would be consistent with the observed activation energy of $0.80 \mathrm{eV}$. One cannot exclude, however, the possibility that the calculated value for the sum of $E_{\text {diff }}$ and 
$E_{\text {form }}$ is higher than the true value. Hence, we cannot completely rule out the adatom mechanism, solely based on the agreement of the calculated numbers with the experiment. If, however, GGA would overestimate the energies, it should do so for both the vacancy and the adatom diffusion, leaving the vacancy mechanism as the one with the lower activation energy, hence as the more effective one. In considering all the facts, the existence of an attachment barrier, the better agreement of the observed activation energy with the activation energy calculated for the vacancy mechanism and the higher activation energy calculated for the adatom mechanism, the evidence is clearly on the side of the vacancy mechanism. In summary, we therefore conclude that the decay of $2 \mathrm{D}$ islands on the $\mathrm{Cu}(100)$ surfaces is via vacancies rather than adatoms as the diffusing species.

This work was supported by grants from the Natural Sciences and Engineering Research Council (NSERC) of Canada and the "Fonds pour la Formation de Chercheurs et l'Aide à la Recherche" (FCAR) of the province of Québec. One of us (G.B.) is thankful to NSERC and FCAR for financial support. Part of the work reported here has been performed on the IBM/SP-2 from CACPUS ("Centre d'Applications du Calcul Paralléle de l'Université de Sherbrooke"). The work was also partially supported by the "Fond der Chemischen Industrie," Germany.
*Present address: Sandia National Laboratories, Mail Stop 1413, Albuquerque, NM 87185-1413.

†Author to whom correspondence should be addressed. Electronic address: h.ibach@fz-juelich.de

${ }^{1}$ M. Zinke-Allmang, L. C. Feldman, and M. H. Grabow, Surf. Sci. Rep. 16, 377 (1992).

${ }^{2}$ K. Morgenstern, G. Rosenfeld, B. Poelsema, and G. Comsa, Phys. Rev. Lett. 74, 2058 (1995).

${ }^{3}$ J. M. Soler, Phys. Rev. B 53, R10 540 (1996).

${ }^{4}$ S. V. Khare and T. L. Einstein, Phys. Rev. B 54, 11752 (1996).

${ }^{5}$ N. C. Bartelt, J. L. Goldberg, T. L. Einstein, E. D. Williams, J. C. Heyraud, and J. J. Métois, Phys. Rev. B 48, 15453 (1993).

${ }^{6}$ M. Giesen, J. Frohn, M. Poensgen, J. F. Wolf, and H. Ibach, J. Vac. Sci. Technol. A 10, 2597 (1992).

${ }^{7}$ M. Giesen-Seibert, F. Schmitz, R. Jentjens, and H. Ibach, Surf. Sci. 329, 47 (1995).

${ }^{8}$ M. C. Bartelt and J. W. Evans, Surf. Sci. 298, 421 (1993).

${ }^{9}$ W. W. Pai, A. K. Swan, Z. Zhang, and J. F. Wendelken, Phys. Rev. Lett. 79, 3210 (1997).

${ }^{10}$ J.-M. Wen, S.-L. Chang, J. W. Brunett, J. W. Evans, and P. A. Thiel, Phys. Rev. Lett. 73, 2591 (1994).

${ }^{11}$ J. B. Hannon, C. Klünker, M. Giesen, G. S. Icking-Konert, H. Ibach, N. C. Bartelt, and J. C. Hamilton, Phys. Rev. Lett. 79, 2506 (1997).
${ }^{12}$ G. Boisvert and L. J. Lewis, Phys. Rev. B 56, 7643 (1997).

${ }^{13}$ J. Frohn, J. F. Wolf, K. Besocke, and M. Teske, Rev. Sci. Instrum. 60, 1200 (1989).

${ }^{14}$ G. Schulze Icking-Konert, M. Giesen, and H. Ibach, Surf. Sci. 398, 37 (1998).

${ }^{15}$ C. Rottman and M. Wortis, Phys. Rev. B 24, 6274 (1981).

${ }^{16}$ M. Giesen-Seibert and H. Ibach, Surf. Sci. 316, 205 (1994).

${ }^{17}$ S. M. Foiles, M. I. Baskes, and M. S. Daw, Phys. Rev. B 33, 7983 (1986).

${ }^{18}$ W. Kohn and L. J. Sham, Phys. Rev. 140, A1133 (1965).

${ }^{19}$ M. C. Payne, M. P. Teter, D. C. Allan, T. A. Arias, and J. D. Joannopoulos, Rev. Mod. Phys. 64, 1045 (1992).

${ }^{20}$ N. Troullier and J. L. Martins, Phys. Rev. B 43, 1993 (1991).

${ }^{21}$ D. M. Ceperley and B. J. Alder, Phys. Rev. Lett. 45, 566 (1980).

${ }^{22}$ J. Perdew and A. Zunger, Phys. Rev. B 23, 5048 (1981).

${ }^{23}$ J. P. Perdew, J. A. Chevary, S. H. Vosko, K. A. Jackson, M. R. Pederson, D. J. Singh, and C. Fiolhas, Phys. Rev. B 46, 6671 (1992).

${ }^{24}$ A. Khein, D. J. Singh, and C. J. Umrigar, Phys. Rev. B 51, 4105 (1995).

${ }^{25}$ P. H. Philipsen and E. J. Baerends, Phys. Rev. B 54, 5326 (1996).

${ }^{26}$ H. Dürr, J. F. Wendelken, and J.-K. Zuo, Surf. Sci. 328, L527 (1995) and references therein.

${ }^{27}$ M. Karimi, T. Tomkowski, G. Vidali, and O. Biham, Phys. Rev. B 52, 5364 (1995). 